\title{
An Overview of the upcoming HomePlug AV Standard
}

\author{
Kaywan H. Afkhamie, Srinivas Katar, Larry Yonge \\ $R \& D$ Department \\ Intellon Corporation \\ Ocala, FL, USA \\ $\{$ kaywan.afkhamie, srinivas.katar, \\ larry.yonge\}@intellon.com
}

\author{
Richard Newman \\ Dept. of Computer and Information Science and Eng. \\ University of Florida \\ Gainesville, FL, USA \\ nemo@cise.ufl.edu
}

\begin{abstract}
In 2000, the HomePlug organization has developed the HomePlug 1.0 standard which became the basis for the world's most widely deployed power line communication system. Now the organization is undertaking the task of developing the specifications for a second generation technology, HomePlug AV, which will be geared towards the distribution of voice, video, and data throughout the home, once again over the power line medium. This specification is targeted for release in the second quarter of 2005.

In this paper, we give an introduction to some of the basic elements of the MAC and PHY layer of the HomePlug AV specification. We describe how some of the system level choices are influenced by either the characteristics of the power line channel, by the regulatory environment, by application requirements, or by implementation constraints. Finally, we discuss the performance of the proposed system measured with prototypes of the proposed system in typical North American homes.
\end{abstract}

Keywords-Standards and Regulations; System Architectures; OFDM Techniques;

\section{INTRODUCTION}

HomePlug AV will be the second major standard released by the HomePlug Powerline Alliance. It follows the HomePlug 1.0 standard of 2000, which has been widely deployed in the market, and is used internationally by home networking equipment manufacturers. While HomePlug 1.0 was designed mainly to distribute broadband internet access in the home, it is HomePlug AV's objective to distribute Audio/Video content within the house, as well as data.

The nature of these target applications brings with itself a new, more stringent set of requirements: higher data rates are required to provide support for multiple HDTV streams, Quality of Service metrics must be met in terms of latency, jitter, and very low frame error rates (for video and voice applications), and since many target applications require embedding of the communications circuitry in consumer electronics equipment, the add-in cost must be as low as possible. Table 1 lists some bandwidth and QoS requirements for popular AV applications.

\begin{tabular}{|l|l|l|l|l|}
\hline Application & $\begin{array}{l}\text { Bandwidth } \\
\text { (Mbps) }\end{array}$ & $\begin{array}{l}\text { Latency } \\
\text { (msec) }\end{array}$ & $\begin{array}{l}\text { Jitter } \\
\text { (nsec) }\end{array}$ & $\begin{array}{l}\text { PLP } \\
\text { (log) }\end{array}$ \\
\hline HDTV & 25 & 300 & 500 & -10 \\
\hline SDTV & 4 & 300 & 500 & -10 \\
\hline DVD & 6 & 300 & 500 & -10 \\
\hline VOIP & 64 & 10 & 10000 & -2 \\
\hline Gaming & 0.1 & 10 & N/A & -6 \\
\hline Video conf. & 1 & 75 & 10000 & -6 \\
\hline
\end{tabular}

Table 1 - Typical Multimedia QoS Requirements

The last requirement for low chip costs is further emphasized by the competitive nature of the home networking market. Powerline communications is only one of several technologies available. Alternative technologies include the suite of WiFi standards, which have the obvious advantage of mobility, regular Ethernet networking which is being built into more and more homes, and other evolving systems such as the MoCA standard for home networking over coax, and Ultrawideband (UWB) communications for high data rate short range communications.

Although these technologies appear to be capable of providing the basis for such multimedia communication, a careful examination reveals several possible deficiencies. For example, the popular IEEE 802.x suite of protocols (including the emerging IEEE 802.n standard) does not provide complete house coverage (with a single access point) at adequate data rates and reliability to provide a robust multimedia solution. While the new proposed technologies for the UWB standard will certainly have adequate bandwidth, their reach will likely be confined to a single room rather than the entire home. The MoCA standard, while possibly offering a solution for video distribution between video sources and players already connected to the exiting coaxial video cabling, fails to offer whole house coverage for other applications such as audio and VOIP, since video cabling is typically limited. Phone line networks also have limited phone connections inside the home.

A further requirement imposed on the HomePlugAV standard is that it's devices should coexist with existing devices 
built to the HomePlug 1.0 standard and also with devices planned to provide broadband access to the home over the low and medium voltage power lines.

Work on the HomePlugAV standard commenced in the summer of 2003, when the alliance detailed the above requirements in a Marketing Requirements Document and a subsequent request for proposals was issued. Technology evaluation started in the fall of 2003 and was completed in the second quarter of 2004. The eventual selection pieced together the best parts of multiple proposals. The final specifications document is expected to be released in the second quarter of 2005.

In sections 2 and 3 of the paper we discuss in more detail some of the basic choices made in HomePlug AV's PHY and MAC layer specifications. The choices typically reflect some of the marketing requirements as detailed above in this section, or requirements imposed by the power line medium or the regulatory environment. In Section 4, we show some performance results obtained with early prototypes.

\section{PHY LAYER}

\section{A. The Power Line Channel}

The power line channel between two nodes in a home is generally characterized by (1) attenuation, (2) multipath, (3) time variations, and (4) noise. Each of these characteristics affect design choices in their own way.

- Due to possibly high attenuation (often up to approx. $60 \mathrm{~dB})$ there is a hard to overcome near-far effect, which rules out a CDMA based system, and makes it hard to implement echo cancellation.

- The impedance mismatches at various points in the power line network cause reflections and multipath, with average delay spreads of between 1 and $2 \mu$ sec. The frequency selective spectrum of such a power line channel favors the use of OFDM as it allows precise frequency domain adaptation of the transmitted waveform, and also allows easy mitigation (via insertion of a guard interval) of the delay spread with little overhead provided a long enough symbol length.

- While the power line channel can be modeled as timeinvariant compared to the length of a typical packet (1$2 \mathrm{~ms}$ ), time-variations do occur on the power line channel in two distinct ways that affect channel adaptation. Long-term changes occur when electrical devices are connected or disconnected from an outlet, causing step changes in the impulse response characteristics, and some periodic channel variation is common with the $60 \mathrm{~Hz}$ line cycle due to switching power supplies, or dimmer switches causing periodic impulsive spikes. To transmit data as close as possible to capacity, HomePlugAV transmitters adapt their signaling depending on fluctuations within the line cycle.
- The harshness of noise on the power line channel is already well documented. HomePlug AV combats impulsive noises and narrow band noises with dedicated receive side signal processing.

Figures 1 through 3 each show snapshots of signal and noise on the power line. They illustrate the severity of the noise, especially when causing large time domain impulses, and the fact that the noise in many cases changes characteristics on different parts of the line cycle.

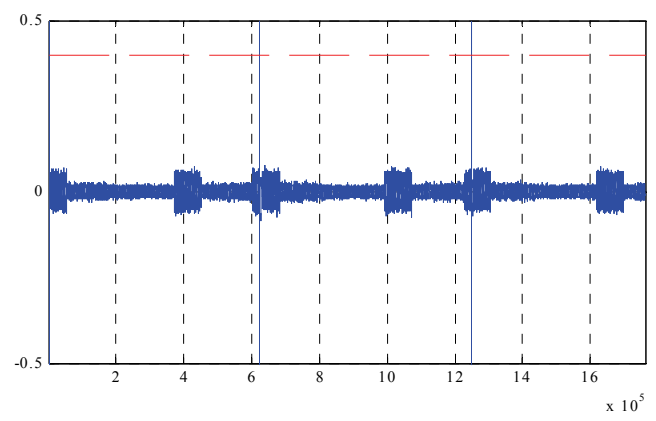

Figure 1. AWGN Channel with Halogen Impairment

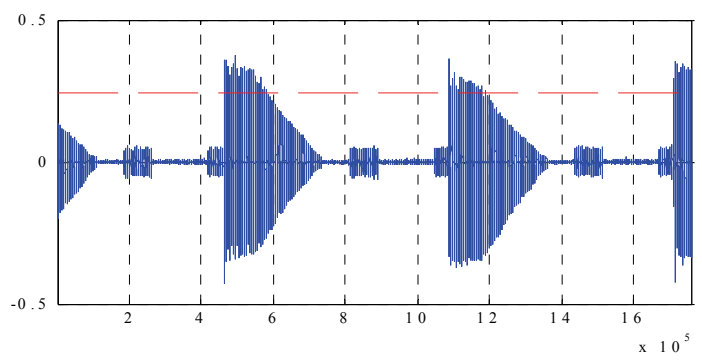

Figure 2. AWGN Channel with Dimmer Switch Impairment

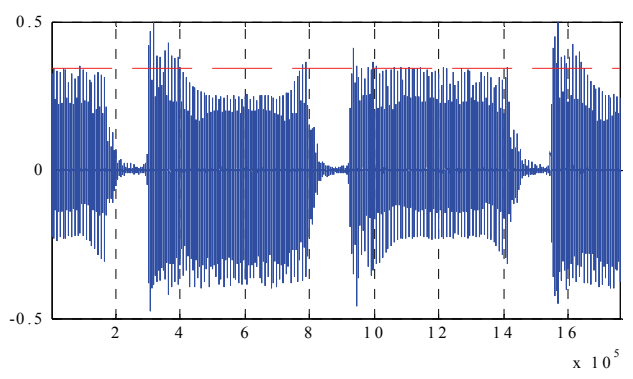

Figure 3. AWGN Channel with Switching Power Supply Impairment

\section{B. The Regulatory Environment}

In North America, FCC regulations limit the frequency range for power line transmissions to $1.8-30 \mathrm{MHz}$, and the existence of amateur radio bands causes additional gaps in this 
frequency range. In other parts of the world regulatory bodies impose on power line communication systems to have an adaptable transmit spectrum. In conjunction, these restrictions led to the adoption of a long OFDM symbol length for HomePlug AV. HomePlug AV employs a 3072 point FFT with 1536 carrier spaced in intervals of $24.414 \mathrm{kHz}$ from DC to $37.5 \mathrm{MHz}$. This creates an OFDM symbol length of $40.96 \mu \mathrm{sec}$, which is long enough so that the symbol can undergo timedomain shaping without the addition of too much overhead, and without severe performance degradation. Time-domain pulse-shaping is applied to OFDM symbols to contain their frequency shape much more than the typical $\sin (x) / x$, thus frequency notches can be created in the transmit PSD by merely turning off carriers rather than using transmit notch filters. This is illustrated in Figure 4 where we show the default transmit PSD mask for operating a HomePlug AV system in North America - the frequency notches correspond to the amateur radio bands.

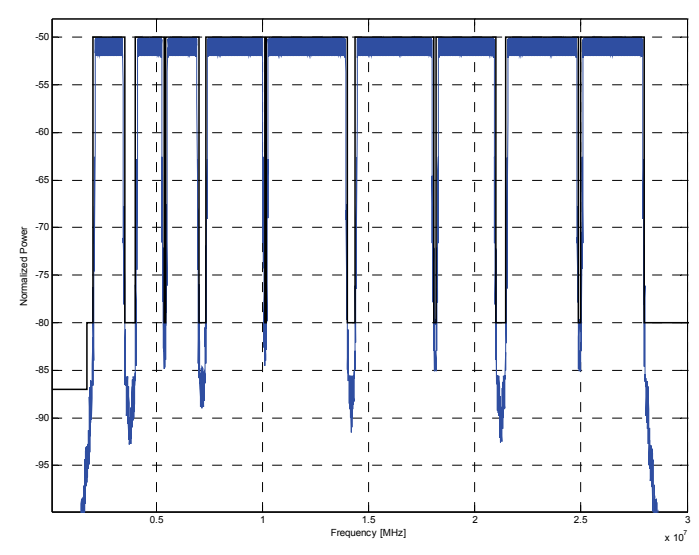

Figure 4. Transmit PSD Mask - North America

\section{Further PHY Layer Features of HomePlug AV}

In addition to the design choices discussed in Sections 2.1 and 2.2, HomePlug AV employs a number of additional features to achieve the higher throughputs required in the targeted set of applications.

- Turbo codes with varying code rates are employed to gain additional resilience to noisy environments, and increase coding gain over the concatenated codes of HomePlug 1.0.

- Coherent modulation is supported, and modulation types include BPSK, QPSK, 8-QAM, 16-QAM, 64QAM, 256-QAM and 1024-QAM

- To enable coexistence between HomePlug 1.0 devices and HomePlug AV devices, the HomePlug AV PHY is equipped with the ability to send and receive HomePlug 1.0 frame control signals, and it utilizes a similar synchronization scheme (all devices thus have the ability to detect packets of either system).
- Several robust modes of operation are provided in HomePlug AV for the communication of network synchronization information, for session setup, multicast and broadcast modes. For example, a 10 Mbps broadcast mode can be employed on the vast majority (99\%) of all power line channels without the requirement of exchanging channel information between transmitter and receiver.

- Channel Estimation is a critical element of the HomePlug AV system. In addition to the line cycle synchronization ability discussed above, HomePlug AV employs higher order modulations up to 1024 QAM, supports variable bit-loading (each carrier individually selects modulation type according to the SNR it observes), monitors carrier signal-to-noise ratios without the explicit need for channel estimation frames (estimates SNR on bit-loaded packets), and is able to track changes in the channel with high efficiency $(93 \%$ of ideal, i.e. a priori channel and noise information).

\section{MAC LAYER}

The Medium Access Control (MAC) layer in high-speed power line communication cannot use the same approaches used in lower-speed PLC. The overhead per MPDU is fixed, and is an increasing impediment to efficiency as the PHY data rate increases. Furthermore, regardless of the channel adaptation, there are likely to be errors due to impulse noise that damage portions of MPDUs beyond repair. These qualities call for a more sophisticated MAC framing approach, one that allows concatenation of multiple MAC Service Data Units (MSDUs) and that also allows efficient retransmission of the damaged parts of an MPDU.

HomePlug AV will use a two-level MAC framing scheme. Incoming MSDUs are packaged with minimal overhead into MAC frames, which then form a MAC frame stream. This is treated as a byte stream by the segmentation process, which forms fixed-size segments for reliable transmission. Each segment is given a header and trailer that allow it to be encrypted, sent, decrypted, and delivered independently as a PHY Block (PB). The PB header contains sequence numbers that support reassembly of the original MAC frame stream from segments delivered out of order. It also contains information that allows MAC frame resynchronization after a portion of the MAC frame stream is lost. Each PB is sent in its own FEC block, and the PB check sequence allows uncorrectable FEC blocks to be detected reliably. Each PB can be individually acknowledged using a selective acknowledgement response, and only the damaged PBs are retransmitted. This results in a highly efficient framing and error correction scheme (illustrated in Figure 5).

Beyond these efficiency concerns, multimedia applications that will use HomePlug AV have stringent requirements on latency, jitter, and packet loss tolerance. Jitter requirements are met by synchronizing the clocks of the sender and the receiver, 

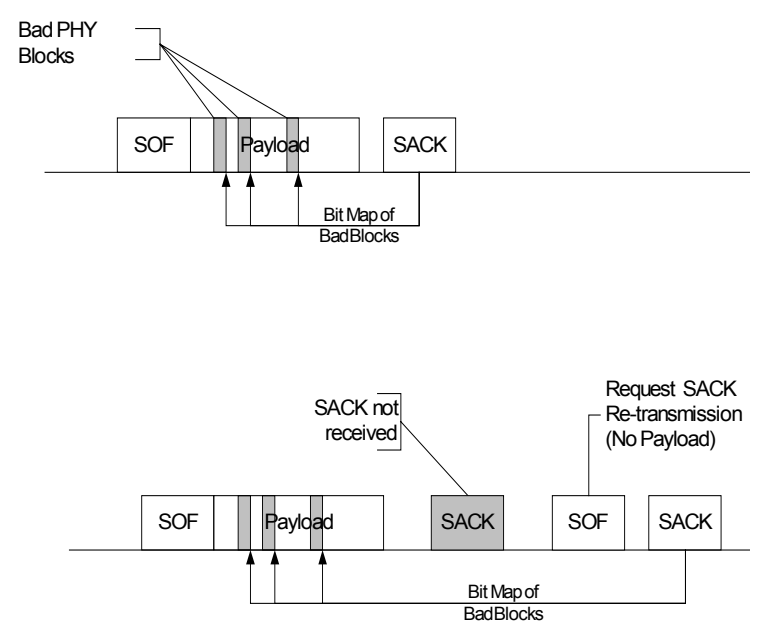

Figure 5. Top: Normal SACK transmission, Bottom: Lost SACK and retransmission of SACK

and using timestamps on the MSDUs. However, meeting latency and packet loss requirements are greater challenges.

Since there is no continuous physical carrier sense, the nodes must maintain virtual carrier sense (VCS). Collisions are very costly, as they cause nodes to lose VCS. For reliability and for efficiency reasons, HomePlug AV uses a centrally scheduled, beacon-based approach. This allows streams to be scheduled at the same part of the line cycle, and so they are able to adapt very well to the channel. With periodic beacons, access allocations can be scheduled so that each stream has a sufficient number of transmission attempts to meet the latency and error rate requirements.

A stream with QoS requirements must first establish a connection with the Central Coordinator (CCo), which makes admission control decisions. The CCo periodically issues beacon frames containing scheduling information that allocates time to each connection and to CSMA/CA traffic as well. Allocations may be persistent or non-persistent, with a common persistence value for all persistent allocations. Impending persistent allocations are also advertised, along with the period in which they become effective. This mechanism allows stations to miss the beacon now and then without suffering for it.

Since the source rate may change, and the PHY rate may change, a connection needs to be able to modify its allocation. The persistent allocation mechanism is reliable, but cannot respond very quickly, so non-persistent allocations are also supported. These allow the CCo to respond within one beacon period to urgent needs of connections.

CSMA/CA access similar to HomePlug 1.0 is also supported, and is intended for control traffic and connectionless traffic. Figures 6 and 7 illustrate the overall access scheme and the allocation of the contention free or TDMA region to separate links.

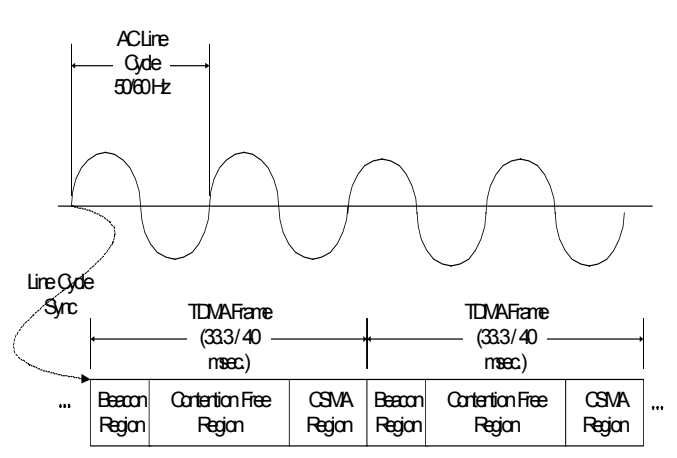

Figure 6. HomePlug AV Access Control

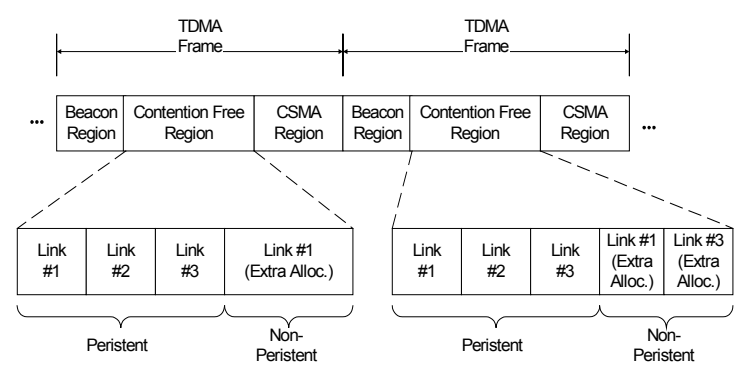

Figure 7. HomePlug AV Scheduling

\section{System Performance COMPARED to HomePlug 1.0}

Experimental systems of HomePlugAV have been field tested in houses, suggesting that on average a HomePlug AV system achieves 10 times the data rate of a 1.0 system. For example, the average PHY layer data rate measured in a large number of homes and a large number of outlet pairs per home is approximately $85 \mathrm{Mbps}$. The increase is due (approximately) to the following factors.

- More Bandwidth (50\% more used bandwidth)

- More Power (Transmit PSD is $2.2 \mathrm{~dB}$ higher, due to lower peak-to-average ratio)

- Coherent transmission (improves $\sim 2.5 \mathrm{~dB}$ over differential transmission in HP1.0)

- Higher Order Modulation (modulates up to 1024QAM, taking advantage of higher SNRs when available)

- Powerful Forward Error Correction (Turbo Codes have about $2.5 \mathrm{~dB}$ advantage over concatenated codes of HP1.0)

- Smart Channel Adaptation (full variable bit-loading, unsolicited channel information updates, line cycle adaptation)

- Efficient Signaling (minimal framing overhead, minimal cyclic prefix)

- Additional Signal Processing 


\section{REFERENCES}

[1] Yu-ju Lin, Haniph A. Latchman, Minkyu Lee and Srinivas Katar, "Power line Communication Network Infrastructure For Smart Homes", IEEE Wireless Communications, Volume 9, Issue 6, Pages: 104-111, December, 2002.

[2] HomePlug Powerline Alliance, http://www.homeplug.org [February 10, 2005]

[3] M.K. Lee, , R. Newman, H.A. Latchman, S. Katar, and L. Yonge, "HomePlug 1.0 Powerline Communication LANs -Protocol Description and Comparative Performance Results", accepted for publication in the Special Issue of the International Journal on Communication Systems on Powerline Communications, pages 447-473, May, 2003

[4] Y. Lin, H. Latchman, S. Katar, and M.K. Lee", "Theoretical and Field Performance Comparison between 802.11 Wireless and Powerline HomePlug 1.0 Protocols" accepted for publication in the IEEE Communications Magazine with Focus Theme on Powerline Local Area Networks, pages 54-63, May, 2003.

[5] Current Technologies http://www.currenttechnologies.com

[6] Amperion Corporation, http://www.amperion.com

[7] Ambient Corporation February 2005, http://www.ambientcorp.com

[8] Baowei Ji, Archana Rao, Minkyu Lee, Haniph A. Latchman, Srinivas Katar, "Multimedia in Home Networking" - CITSA 2004 / ISAS 2004 Proceedings Volume 1, Network Technologies, Page Nos: 397-404.

[9] Barnes, J.S., “A Physical Multipath Model For Powerline Channels at High Frequencies", Procceedings of the International Symposium on Powerline Communication and Its Applications, 1998, 76-89.

[10] Minkyu Lee,Haniph A. Latchman, Richard E. Newman, Srinivas Katar, and Larry Yonge, "Field performance comparison of IEEE $802.11 \mathrm{~b}$ and HomePlug 1.0", 27th Annual IEEE Conference on Local Computer Networks, Pages: 598-599, 2002. 